\title{
Pneumococcal Carriage and Antibiotic \\ Susceptibility Patterns in Mother-Baby Pairs in a rural community in Eastern Uganda
}

\section{Gabriel Madut Akech}

Busitema University, Faculty of Health Sciences

\section{Mercy Naloli}

Busitema University Faculty of Health Sciences

\section{Paul Sebwami}

Busitema University Faculty of Health Sciences

\section{Patrick Kazibwe}

Busitema University Faculty of Health Sciences

\section{Maureen Atwikiriize}

Busitema University Faculty of Health Sciences

\section{Julius Onyait}

Ngora Health Center IV

\section{Paul Oboth}

Busitema University Faculty of Health Sciences

\section{Julius Nteziyaremye}

Busitema University Faculty of Health Sciences

\section{Rebecca Nekaka}

Busitema University Faculty of Health Sciences Jacob Stanley Iramiot ( $\sim$ jiramiot@gmail.com )

Busitema University-Mbale Campus https://orcid.org/0000-0002-9239-6641

\section{Research note}

Keywords: Pneumococcal carriage, mother-baby pair, antibiotic susceptibility pattern, immunization with PCV 10, Eastern Uganda

Posted Date: December 3rd, 2019

DOI: https://doi.org/10.21203/rs.2.18142/v1

License: (c) (1) This work is licensed under a Creative Commons Attribution 4.0 International License. Read Full License 


\section{Abstract}

Objective: This study aimed to estimate pneumococcal carriage and determine antibiotic susceptibility patterns of the pneumococci isolated in the mother-baby pairs in Ngora district after the roll out of the pneumococcal vaccine. We hypothesized that high carriage of S. pneumoniae in mothers leads to carriage in their babies and hence a greater chance of contacting pneumoniae.

Results: Consecutive sampling technique was used to select 152 mother-baby pairs from the community visits and those seeking care at the health facility. We collected nasal swabs from both baby and mother for culture and sensitivity using the Kirby-Bauer's agar disc diffusion method. This study found that there was a low prevalence of pneumococcal carriage in the mother-baby pair in Ngora district. We also observed high rates microbial resistance to Penicillin which is the first-line management of pneumonia in Uganda. The relationship between pneumococcal carriage and immunization status suggest that Pneumococcal vaccine is protective against pneumococcal carriage. Resistance of S. pneumoniae to the commonly used antibiotics was high. Key words: Pneumococcal carriage, mother-baby pair, antibiotic susceptibility pattern, immunization with PCV 10, Eastern Uganda.

\section{Methods}

\section{Research design and setting}

A cross-sectional study was carried out in Ngora district Health Centre IV which serves approximately a population of 142,487. Ngora district is one of the districts in Teso sub region curved out of Kumi district in the year 2010 by the Uganda parliamentary act. Ngora district covers an area of approximately 715.9 square kilometers and predominantly inhabited by the Iteso and Kumam ethnicities. According to the Uganda health care hierarchy of organization, a Health Center IV (HCIV) is expected to serve a population of up to 100,000 people meaning at its current capacity, this health facility operates above the level of a Health center IV [10].

\section{Specimen collection and transport}

Nasal pharyngeal specimens were collected from the posterior nasopharynx of the mother and the baby using sterile cotton swab sticks moistened with $0.9 \%$ physiological saline. Separate swabs were used to collect samples from a mother and a baby in a mother-baby pair. To control sample contamination, the swab was placed in a casing containing Amies transport medium and immediately placed into the cool box containing ice packs for transportation to Busitema University Microbiology laboratory for culture and susceptibility testing within 12 hours. For this study, we defined a baby as any person under the age of 5 years and a mother was considered as either biological or any other female in direct care of the baby.

\section{Laboratory Procedures}


Samples were cultured on sheep Blood agar and chocolate agar followed by 24 hours of incubation at $37^{\circ} \mathrm{C}$ anaerobically. The isolates were identified morphologically by colonal appearance and gram staining. Optochin sensitivity and bile solubility testing were conducted on colonies that were potentially identifiable as $S$. pneumoniae by alpha-haemolytic appearance on the culture media and lancet shaped gram positive cocci appearing in pairs.

A 0.5 McFaland standard of S. pneumoniae was made from a 24-hour subculture by suspending colonies in sterile normal saline and inoculated by swabbing onto a plate of Mueller Hinton Agar supplemented with $7 \%$ sheep blood for susceptibility testing. Antibiotic susceptibility to Penicillin G (1U), Chloramphenicol $(30 \mu \mathrm{g})$, Tetracycline $(10 \mu \mathrm{g})$, Clindamycin $(2 \mu \mathrm{g})$, Erythromycin $(30 \mu \mathrm{g})$ and Ceftriaxone $(30 \mu \mathrm{g})$ was determined using modified Kirby-Bauer's agar disc diffusion methods and the disc zone diameters were interpreted using the Clinical and Laboratory Standards Institute Guidelines.

\section{Data analysis}

Collected data was entered in Microsoft excel, cleaned, coded and imported to SPSS Version 16.0 statistical package for analysis. Statistical frequency distribution tables and graphs were used for data presentation in terms of proportions, absolute values, percentages and confidence intervals for point approximations at $95 \%$ level of confidence with a $P<0.05$ considered as statistically important.

\section{Results}

\section{Demographic characteristics of the study participants}

The study participants comprised of 152 mothers and 152 babies. Of the 152 babies, 74 were male and 78 were female with the age range of 0-70 months. The youngest mother was 16 years whereas the oldest was 44 years. None of the mothers who participated in the study reported having formal employment.

\section{Prevalence of Pneumococci in Mother-Baby Pairs.}

During the study, 304 samples were collected, 152 from the mothers and 152 from the children making 152 mother-baby pairs. All samples were cultured and antibiotic susceptibility was carried out on the isolated pneumococci. Out of 152 samples from the mothers only five $(5 / 152)$ isolates of pneumococci were obtained whereas seven (7/152) isolated from the babies. Only one mother-baby pair (1/152) was found to be colonized with pneumococci in both mother and baby and the rest of $S$. pneumoniae colonized either the mother or baby. 


\section{Immunization coverage}

During data collection, immunization status of the baby was categorized in the following divisions; fully immunized, not immunized and partially immunized in the age-groups (Fig. 1). There was high immunization coverage among the children above 12 months old but lower in the $3.5-<12$ age group.

\section{Antibiogram}

\section{The antibiotic susceptibility testing was done on both positive isolates for mother and baby (Table 1).}

Table 1: Antibiotic susceptibility patterns of $S$. pneumoniae to the commonly used antibiotics

\begin{tabular}{llll} 
Drugs & Sensitive & Intermediate & Resistant \\
\hline Penicillin G & $6(50)$ & $3(25)$ & $3(25)$ \\
\hline Chloramphenicol & $6(50)$ & $0(0)$ & $6(50)$ \\
\hline Tetracycline & $4(33)$ & $2(17)$ & $6(50)$ \\
\hline Clindamycin & $9(75)$ & $1(8)$ & $2(17)$ \\
\hline Erythromycin & $8(67)$ & $0(0)$ & $4(33)$ \\
\hline Ceftriaxone & $8(67)$ & $1(8)$ & $3(25)$
\end{tabular}

Generally, high trend of anti-microbial resistance was observed among the $S$. pneumoniae isolated. The highest resistance patterns were recorded with Chloramphenicol (50\%) and Tetracycline (50\%) whereas the lowest resistance was recorded in Clindamycin (17\%).

Factors associated with pneumococcal carriage

Table 2: Factors associated with pneumococcal carriage 


\begin{tabular}{llll} 
Factors Associated with Carriage & Odds Ration & $P$-Value & (95 Cl) \\
\hline Age Group (Months) & 1.3603 & 0.478 & $0.5818-3.1804$ \\
$0-<1.5$ & & & \\
$1.5-<3.5$ & & & \\
$3.5-<12$ & & & \\
$12-<60$ & & & \\
\hline Immunization Status & & 0.010 & $1.5939-32.1791$ \\
\hline Sex of the Babies & 7.1617 & 0.290 & $0.4633-13.1224$ \\
\hline Age of the Mother & 2.4658 & 0.194 & $0.7731-1.0536$ \\
\hline Address & 0.9025 & 0.262 & $0.7753-1.07171$
\end{tabular}

Babies that were fully immunized had a less likelihood to be colonized by $S$. pneumoniae than their non immunized counterparts $P<0.05$. Other factors examined by this study were not significantly associated with colonization with S. pneumoniae among the babies (Table 2).

\section{Discussion}

We determined the prevalence of pneumococcal carriage and factors associated with colonization of pneumococci in a mother-baby pair in our study. Out of the 304 nasal swabs cultured, only $12(3.95 \%)$ were positive for pneumococci, $7(4.61 \%)$ in children below 5 years and $5(3.29 \%)$ in mothers. We report a low carriage of pneumococci among mothers and babies that were included in this study. In contrast, a previous study in Iganga/Mayuge reported high carriage rates of over $50 \%$ in children aged less than 5 years [4]. In the Iganga/Mayuge study participants were selected on the basis of presentation with pneumonia symptoms as defined by WHO guidelines as opposed to our study which included all children that fulfilled our selection criteria which did not include signs and symptoms of pneumonia. In a similar study carried out in Kenya, $90.0 \%$ of children were colonized with pneumococci. Both the Iganga/Mayuge and Kenyan studies were carried out prior to the introduction of PCV10 accounting for the difference in the carriage observed in our study. Different studies have shown varied carriage rates of pneumococci among children below five years in Uganda and elsewhere $[4,11,12]$ with most of them reporting a higher carriage rate than reported in our study. A systematic review reported a carriage rate in Africa to range between $21-94 \%$ [13] with more studies done among children than in adult population. The high immunization coverage for PCV10 in Ngora district could further explain the low carriage rate of pneumococci in our study as opposed to the Iganga/Mayuge study that indicated a high carriage rate of $56 \%$ at a lower immunization coverage of $42 \%$ PCV10 and 54\% PCV13. There was a statistically 
significant relationship between the pneumococcal carriage and immunization status of the babies in our study (Table 2). Pneumococcal carriage is a prerequisite to disease, therefore our findings suggest that full immunization with PCV10 is protective against pneumococcal carriage and hence pneumonia caused by streptococcus pneumoniae. Several studies have reported decrease in the burden of invasive pneumococcal disease and serotype distribution since the introduction of PCV vaccine [14-17]. The immunization coverage for the first dose of PCV10 (PCV1) in Ngora district was 97.78\% (133/136) and $2.22 \%(3 / 136)$ of children above six weeks had not received PCV1. Of the 152 participants $10.53 \%$ [16] were children below six weeks and were therefore not eligible for immunization with PCV10. The immunization coverage for PCV3 was $90.99 \%$ (101/111). Children below fourteen weeks who had not received PCV3 were excluded from the denominator because they were not eligible.

We also report a low carriage rate of pneumococci among the mothers. A similar study in coastal Kenya indicates that pneumococcal carriage was more associated with children below 5 years than the adults [12]. The low carriage rate of pneumococci among adults has been attributed by other studies to development of natural immunity [18]. The upper respiratory tract apparently appears a disadvantageous niche for streptococcus pneumoniae due to development of mucosal host defenses such as slgA $[19,20]$. Also vaccination with PCV has resulted into the development of herd immunity in the adult population against $S$. pneumoniae [12]. Cases of pneumococcal colonization are however reported to rise in the elderly population due to immune senescence [21] with many countries not considering the importance of immunization this group of people.

From our analysis, there was no statistically significant association in risk of carriage of pneumococci with sex/gender of the child. This finding is similar to the results of a systematic review in Africa which noted that there was no association between pneumococcal carriage and gender [13] though one study associated pneumococcal carriage with males and the other that reported association of carriage with females.

A high trend of anti-microbial resistance was observed in Chloramphenicol, Tetracycline and Erythromycin. Other studies have similarly reported high resistance of pneumococci to the commonly used antibiotics $[22,23]$. In a study of erythromycin resistant $S$. pneumoniae $81 \%$ of the isolates were resistant to tetracycline, $76 \%$ were multi-drug resistant whereas $12 \%$ were resistant to clindamycin, tetracycline, chloramphenicol and kanamycin combined [22]. As opposed to our study, low resistance rates against tetracycline, erythromycin, chloramphenicol and Ceftriaxone were reported in Tanzania [23]. Again, contrary to our findings, an earlier study in Uganda reported no resistance against erythromycin and ceftriaxone [24], indicating emergency of antimicrobial resistance against those drugs which may be attributed to the irrational use of antibiotics in Uganda and also due to the fact that such drugs are given empirically since there is no laboratory capacity to carry out culture and sensitivity studies.

\section{Conclusions and recommendations}


We report low pneumococcal carriage in the mother-baby pair in Ngora district. There was no significant relationship between pneumococcal carriage in the mother and prevalence in the baby. The relationship between pneumococcal carriage and immunization status suggest that PCV10 is protective against pneumococcal carriage. Resistance of $S$. pneumoniae to the commonly used antibiotics was high.

\section{Limitations}

We were not able to serotype the pneumococci isolated to determine the circulating serotypes.

\section{Abbreviations}

PCV: Pneumococcal conjugate vaccine, MRRHREC: Mbale Regional Referral Hospital Research and Ethics Committee.

\section{Declarations}

\section{Acknowledgements}

We gratefully acknowledge our research participants who provided useful information for this work. We are also grateful to Dr. Onyait Julius for his role as a preceptor and mentor in this project. Heartfelt Thanks to Rebecca Nekaka for coordinating the whole project.

\section{Funding}

This work was supported by the Department of Community and Public health and the Department of Microbiology and Immunology. The funding only covered only field data collection and laboratory tests.

\section{Availability of data and materials}

All data on which the conclusions of this manuscript are drawn has been made available together with this manuscript.

\section{Author Contributions}

GMA, MN, BW, PS, PK and MA participated in conception of the research idea, study design and drafting the first manuscript; JO, PO and JN conducted critical reviews, RN coordinated the whole project and JSI conducted critical reviews and supervised the study. All authors have read and approved the final version of the manuscript. 


\section{Ethics approval and consent to participate}

We obtained ethical approval for this study from Mbale Regional Referral Hospital Research and Ethics Committee (MRRHREC). A letter of introduction to the study area following approval was obtained from the Busitema University Faculty of Health Sciences community education program office and used by the researchers to seek permission from the Medical superintendent of Ngora district health Centre IV. Written informed consent was obtained preceding to the actual data collection exercise. Access to collected data was restricted to only persons directly involved in the study. Participants were free to withdraw their consent to participate in the study at any stage of the study. Such a decision did not affect the medical care they received or possible participation in future research studies in anyway. The procedures were clearly explained to the research participants. Those who agreed to continue with the study were allowed to consent and participate.

\section{Consent for publication}

Not applicable.

\section{Competing interest}

The authors declare that they have no conflict of interest

\section{Author's details}

${ }^{1}$ Department of Community and Public Health, Faculty of Health Sciences, Busitema University, Mbale, Uganda.

${ }^{2}$ Department of Obstetrics and Gynaecology Faculty of Health Sciences, Busitema University, Mbale, Uganda.

${ }^{3}$ Department of Microbiology and Immunology, Faculty of Health Sciences, Busitema University, Mbale, Uganda.

${ }^{4}$ Ngora Health Center IV, Ngora district local government, Uganda

\section{References}

1. Martins AL, Nascimento D, Schneider IJ, Schuelter-Trevisol F. Incidence of community-acquired infections of lower airways among infants Revista paulista de pediatria : orgao oficial da Sociedade 
de Pediatria de Sao Paulo. 2016;34(2):204-9.

2. Zar HJ, Madhi SA, Aston SJ, Gordon SB. Pneumonia in low and middle income countries: progress and challenges. Thorax. 2013;68(11):1052-6.

3. Sigauge B, Verani JR, Vubil D, Quinto L, Acacio S, Mandomando I, et al. Burden of invasive pneumococcal disease among children in rural Mozambique: 2001-2012. PloS One. 2018;13(1):e0190687.

4. Lindstrand A, Kalyango J, AlfveÂa T, Kadobera D, Bwanga F, Stefan P, et al. Pneumococal Carriage in Children under Five years in Uganda- Will the present pneumococal conjugate vaccine be appropriate? PLoS ONE. 2016;11(11):e0166018.

5. Adler N, Weber H, Gunadasa I, N. F. Aherence to therapeutic guidelines for patients with communityaquired pneumonia in Australian hospitals. Clinical medicine insights, circulatory, respiratory and pulmonary medicine. 2014;8:17-20.

6. Nair GB, Niederman MS. Nosocomial Pneumonia. Lessons Learned. Critical Care Clinics. 2013;29(3):521-46.

7. Le Roux DM, Zar HJ. Community-acquired pneumonia in children - a changing spectrum of disease. Pediatric radiology. 2017;47(11):1392-8.

8. levine O, Dinleyci E C. Pneumonia: The forgotten killer of children. Annotated. 2010;4(10):27-8.

9. Green RJ, Kolberg JM. Neonatal pneumonia in sub-Saharan Africa. Pneumonia. 2016;8(1):3.

10. Ahebwa Amelia., Akol Walter., Achong Emmanuel., Mugerwa Timothy., Nakayenga Aminah., Omega Philip., et al. Awareness of antimicrobial resistance among Primary Health Care workers in Buyende district, rural eastern Uganda. Microbiology Research Journal International. 2017;22(5):1-11.

11. Rutebemberwa E, Mpeka B, Pariyo G, Peterson S, Mworozi E, Bwanga F, et al. High prevalence of antibiotic resistance in nasopharyngeal bacterial isolates from healthy children in rural Uganda: A cross sectional study. Upsala Journal of Medical Sciences. 2015;120(4):249-56.

12. Abdullahi O, Nyiro J, Lewa P, Slack M, M. S. The descriptive epidemiology of Streptococcus pneumoniae ans Haemophilus influenzae nasopharyngeal carriage in children and adults in Kilifi district, Kenya. Pediatr Infect Dis J. 2008;27:59-64.

13. Usuf E, Bottomley C, Adegbola RA, Hall A. Pneumococcal Carriage in Sub-Saharan Africa-A systematic Review. PLoS ONE. 2014;9(1):e85001.

14. Guevara M, Barricarte A, Gil-Setas A, García-Irure JJ, Beristain X, Torroba L, et al. Changing epidemiology of invasive pneumococcal disease following increased coverage with the heptavalent conjugate vaccine in Navarre, Spain. Clinical Microbiology and Infection. 2009;15(11):1013-9.

15. Kellner J. Update on the access of the pneumococcal conjugate vaccine. Paediatrics and child health. 2011;16(14):233-40.

16. Williams SR, Mernagh PJ, Lee MHT, Tan JT. Changing epidemiology of invasive pneumococcal disease in Australian children after introduction of a 7-valent pneumococcal conjugate vaccine. Medical Journal of Australia. 2011;194(3):116-20. 
17. Ho P-L, Chiu SS, Ang I, Lau Y-L. Serotypes and antimicrobial susceptibilities of invasive Streptococcus pneumoniae before and after introduction of 7-valent pneumococcal conjugate vaccine, Hong Kong, 1995-2009. Vaccine. 2011;29(17):3270-5.

18. Ramos-Sevillano E, Ercoli G, Brown JS. Mechanisms of Naturally Acquired Immunity to Streptococcus pneumoniae. Frontiers in Immunology. 2019;10(358).

19. Ulrike Binsker., John A. Lees., Alexandria J. Hammond., Weiser. JN. Immune exclusion by naturallyacquired secretory IgA to the pneumococcal pilus-1.

20. Croucher NJ, Campo JJ, Le TQ, Liang X, Bentley SD, Hanage WP, et al. Diverse evolutionary 746 patterns of pneumococcal antigens identified by pangenome-wide immunological screening. 747 Proceeding of the National Academy of Sciences. 2017;114(3):E357.

21. Van Buynder P, Booy R. Pneumococcal vaccination in older persons: where are we today? Pneumonia. 2018;10(1):1.

22. Akdoğan Kittana FN, Mustak IB, Hascelik G, Saricam S, Gurler N, Diker KS. Erythromycin-resistant Streptococcus pneumoniae: phenotypes, genotypes, transposons and pneumococcal vaccine coverage rates. Journal of Medical Microbiology. 2019;68(6):874-81.

23. Moyo SJ, Steinbakk M, Aboud S, Mkopi N, Kasubi M, Blomberg B, et al. Penicillin resistance and serotype distribution of Streptococcus pneumoniae in nasopharyngeal carrier children under 5 years of age in Dar es Salaam, Tanzania. Journal of Medical Microbiology. 2012;61(7):952-9.

24. Joloba ML, Bajaksouzian S, Palavecino E, Whalen C, Jacobs MR. High prevalence of carriage of antibiotic-resistant Streptococcus pneumoniae in children in Kampala Uganda. International Journal of Antimicrobial Agents. 2001;17(5):395-400.

\section{Figures}


Fig.1: PCV 10 Immunization coverage among children below five years

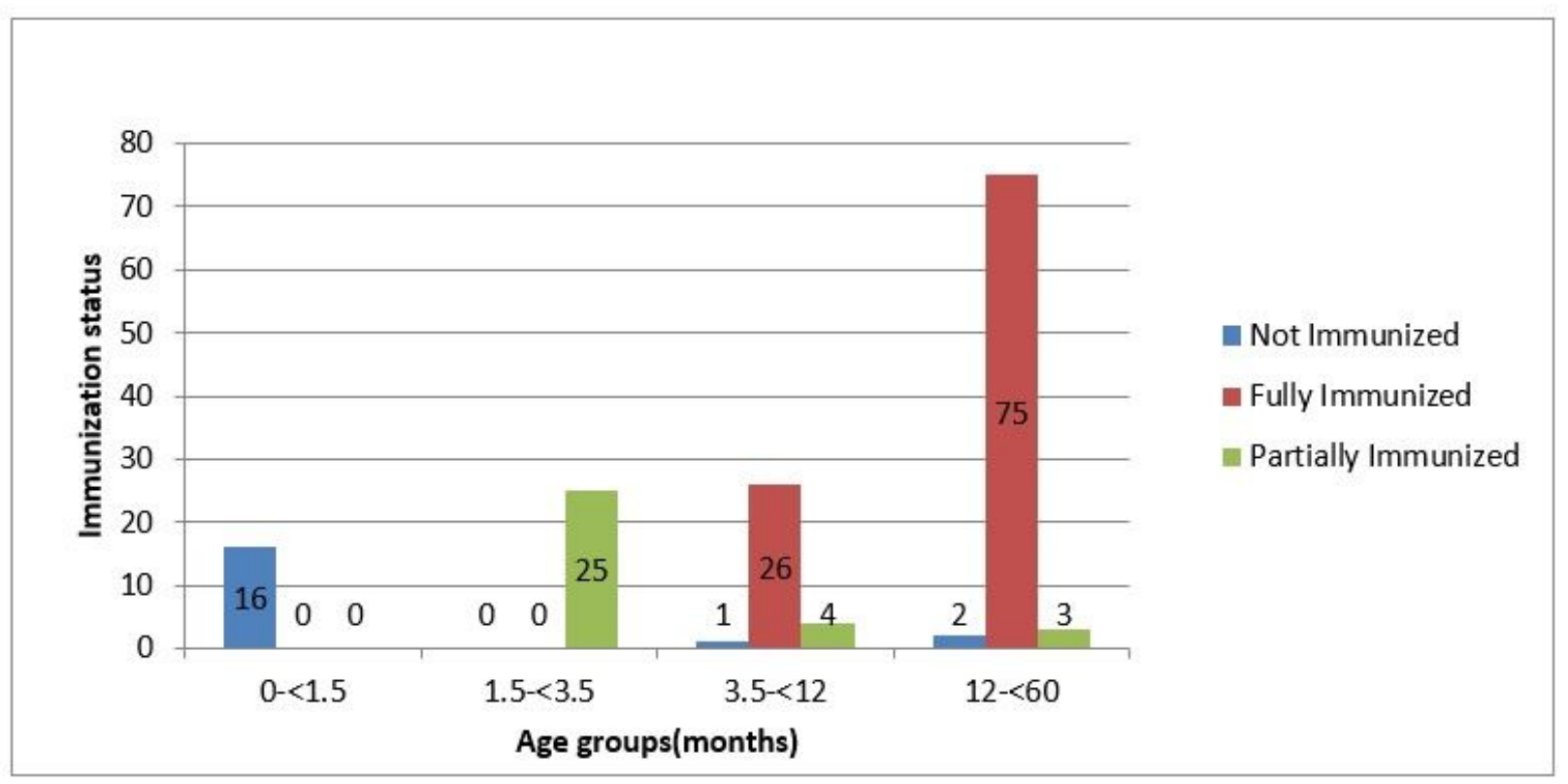

Figure 1

PCV 10 Immunization coverage among children below five years

\section{Supplementary Files}

This is a list of supplementary files associated with this preprint. Click to download.

- suplimentarymaterials28112019.xlsx 